Conference Paper

\title{
Collaborative Artificial Intelligence in Music Production
}

Nicholls, S., Cunningham, S. and Picking, R

This is a paper presented at the Audio Mostly 2018 (AM'18), 12-14 Sept 2018

Copyright of the author(s). Reproduced here with their permission and the permission of the conference organisers.

\section{Recommended citation:}

Nicholls, S., Cunningham, S. and Picking, R (2018) 'Collaborative Artificial Intelligence in Music Production'. In: Proc Audio Mostly 2018 (AM'18), 12-14 Sept 2018, article no. 33. doi: $10.1145 / 3243274.3243311$ 


\section{Collaborative Artificial Intelligence in Music Production}

\author{
Steven Nicholls \\ Wrexham Glyndŵr University \\ Wrexham, UK \\ steve@,drumwithourhands.com
}

\author{
Stuart Cunningham \\ Manchester Metropolitan University \\ Manchester, UK \\ s.cunningham@mmu.ac.uk
}

\author{
Richard Picking \\ Wrexham Glyndŵr University \\ Wrexham, UK \\ r.picking@glyndwr.ac.uk
}

\begin{abstract}
The use of technology has revolutionized the process of music composition, recording, and production in the last 30 years. One fusion of technology and music that has been longstanding is the use of artificial intelligence in the process of music composition. However, much less attention has been given to the application of AI in the process of collaboratively composing and producing a piece of recorded music. The aim of this project is to explore such use of artificial intelligence in music production. The research presented here includes discussion of an auto ethnographic study of the interactions between songwriters, with the intention that these can be used to model the collaborative process and that a computational system could be trained using this information. The research indicated that there were repeated patterns that occurred in relation to the interactions of the participating songwriters.
\end{abstract}

\section{CCS CONCEPTS}

- Applied computing Sound and music computing • Humancentered computing Empirical studies in collaborative and social computing • Computing methodologies Artificial intelligence

\section{KEYWORDS}

Artificial intelligence, collaboration, music production, qualitative.

\section{ACM Reference format:}

Steven Nicholls, Stuart Cunningham and Richard Picking. 2018. Collaborative Artificial Intelligence in Music Production. In Proceedings of Audio Mostly 2018: Sound in Immersion and Emotion (AM'18). ACM, New York, NY, USA, 4 pages. https://doi.org/10.1145/3243274.3243311

\section{Introduction}

Currently, music production has shifted from the use of expensive commercial recording studios, to recording music 'in the box' via computer, without the need for large amounts of outboard equipment and studio space in many cases [1]. This is a pattern that has followed a wider trend in the music industry where, due to the development of the Internet, high quality recorded music has

\footnotetext{
Permission to make digital or hard copies of part or all of this work for personal or classroom use is granted without fee provided that copies are not made or distributed for profit or commercial advantage and that copies bear this notice and the full citation on the first page. Copyrights for third-party components of this work must be honored For all other uses, contact the owner/author(s).

AM'18, September 12-14, 2018, Wrexham, United Kingdom

(C) 2018 Copyright is held by the owner/author(s)

ACM ISBN 978-1-4503-6609-0/18/09.

https://doi.org/10.1145/3243274.3243311
}

become easy to copy and share, and a wider range of music is available to consumers.

The technology available to produce records without professional studio facilities is now so advanced, that in some cases, a commercial record made at home would be indistinguishable from that produced in a professional studio. This means that an artist could take a musical idea from infancy to finished product in a short amount of time, for a relatively small cost, completely without any input from others.

However, the demands of consumers, means that in order for a musical artist to remain relevant and commercially viable, they must produce material at an increasing rate, and therefore should embrace ways to increase productivity.

Historically, song-writing partnerships have been shown to be common practice in popular music for this reason [2]. However, finding a suitable collaborator can be problematic. Currently there are no computer systems that will collaborate with a user, essentially emulating a collaborator.

Previous research in this area examined artificial intelligence (AI) based composition focusing on music generation via a variety of algorithms. A useful example, as it combines three established approaches to AI based composition, is the MAGMA system, which operated by generating notes, based on three researched algorithms [3].

Many compositional systems have been developed in a similar vein, with much of the research focusing on areas such as different ways to generate starting points, or the behaviour of different types of algorithm. The common characteristic in all of these systems is that there is a distinct point at which user interaction ceases and then all work is carried out by the AI system [4]. This would not be the case in a system where the compositional process is conducted between two or more human beings, insomuch as the process is likely to be iterative.

It was proposed that research could be carried out into the nature of collaborative musical partnerships to determine a method for implementing an artificial intelligence-based solution. This could be done by determining key parameters, and defining a set of rules, in the collaborative process and developing a software system to collaborate with a single user to create a number of musical pieces. It was considered that the system could possibly devise a set of fuzzy logic rules based on dialogue, or perhaps even a set of musical data, which had been generated within collaborative interactions. This idea could potentially be expanded to consider collaborative musical production/engineering systems, or musical performance. 


\section{Literature Review}

\subsection{Current Technology and Research}

A review of current technology showed that numerous companies had claimed to have created collaborative AI for the purposes of writing songs. However, in most cases the AI was used to initiate the music on which the human participant builds. Three cases of systems where it was claimed that a collaboration between human beings and AI, or algorithmic processes were reviewed. These were 'Generative Music' [5], 'Amper' [6] and 'Flow Machines' [7].

2.1.1 Generative Music. The term 'generative music' was coined by Brian Eno who defined it as music that was created by a system that was constantly evolving. Music was created by setting up conditions and allowing the system to run autonomously.

Eno's 1996 album 'Generative Music 1' was created using SSEYO Koan, which was a music production program that worked in a similar way to MIDI sequencing, created by Tim and Peter Cole. It operates in conjunction with a range of other SSEYO Koan products. One of these products, the SSEYO Koan Music Engine (SKME), was a real-time music generative music engine, which could be implemented via the Koan system. The SKME is essentially a plugin that is able to generate unpredictable and unrepeatable pieces of music [8].

2.1.2 Amper. Amper was a music composition tool originally designed for creating film soundtracks. The system was based on an algorithm that used characteristics such as 'mood', 'length', and 'style' to create a piece of music. Essentially, Amper was a type of rule-based composition system. The rules were set by the user, and the software was then set in motion. The user then interacted with the software by making all creative decisions based on the ideas that the software had generated. Amper was in this sense, less collaborative, and more of a generative music tool.

2.1.3 Flow Machines. Flow Machines was a project created by Sony Computer Science Laboratories. It was a collaborative compositional project based on the work of Mihalyi Csikszentmihalyi who created the concept of 'Flow States'. A flow state refers to the psychological state that a human being experiences when engaged in certain activities including creative work [9].

Czikczentmihalyi found that a state of 'flow' was experienced by practitioners that had developed a high level of skill and were faced with a high-level challenge in their chosen activity. If the practitioner did not possess a high level of skill, and the chosen activity did not present enough of a challenge, then a flow state did not occur. Similarly, if a practitioner possessed too much or too little skill, in relation to the level of challenge presented, then a flow state would not be experienced.

Flow States would therefore tend to be reported by artists who had developed their own practice to a level where they would be considered to have their own "style" of art. Flow Machines were an algorithmic based collaborative song writing system based on Flow States. Flow Machines work on the principle that a user can improve and develop their own "style" by experimenting using the "styles" of other well-known artists. A database of information based on the famous practitioner is used, in conjunction with an algorithm based on Markov constraints, in order to create a piece of music or literature. In this way, a user can experience writing with the famous practitioner, and essentially develop a style much more quickly than by a natural form of stylistic evolution.

\subsection{The Psychology of Creativity}

Two methods of modelling creativity that were considered to be relevant were the Systems Model of Creativiy by Csikzentmihayli [10] and the 'Four-C Model' developed by Beghetto and Kaufman [11].

2.2.1 The Systems Model of Creativity. In his work on Creativity, Csikszentmihalyi developed what was referred to as a Systems Model of Creativity.

2.2.2 The Four-C Model. The 'Four-C' creativity model, developed by Kaufman and Beghetto, addressed an issue of categorisation of creativity as a concept. Creativity had been divided into two broad categories 'big ' $\mathrm{C}$ '" and 'little 'c"' [12]. 'Big $\mathrm{C}$ ' referring to ideas of such significance that they would be at a level that would be considered culturally impactful; and 'little c' referring to creative ideas processed at a less significant level.

Beghetto and Kaufman attempted to categorise the creative process into four sections with the addition of 'Mini C' and 'Pro C' levels of creativity. The conclusion they reached was that the broad definition of creativity, as 'Big C' and 'little c', was not sufficient to categorize all types of creativity. More specific definitions were considered necessary in order to describe differing degrees and types of creativity.

\section{Auto Ethnographic Study of Song Writing}

It was considered that a series of song-writing sessions should be carried out in order to observe the specific creative behavior of musicians in a collaborative song writing situation. It was expected that an emergent model of collaborative song writing behavior would become apparent. The research approach was as follows:

- Observe the process of collaboration between the researcher and practitioners during two writing and recording sessions;

- Determine practitioners' views on the overall process;

- Ask practitioners to give their opinion on the processes;

- Ask practitioners suggest improvements to the process;

- Identify any relationship between the emergent model of behavior to existing models.

\subsection{Song Writing Sessions (Method)}

A number song writing sessions, each with different musical artists, were carried out. In each session, the artist worked in collaboration with the researcher to write a complete song in a single 8-hour session. In each case the entire process was filmed and later transcribed.

The participants were allowed to take breaks whenever they considered necessary. The time taken to work on the session was not allowed to exceed 8 hours. A song could not be spread over numerous sessions. The aim of the project was to finish a song in a 
single session, or to work on a song until both participants agreed that no more work was able to be done. Participants would be asked to reflect on the process in a questionnaire and the process would be filmed and analyzed at a later date using thematic analysis.

\subsection{Results and Conclusions}

3.2.1 Participant 1. The first participant in the song writing sessions was an acoustic singer songwriter that was part of a professional group that wrote, recorded, and toured extensively.

A basic musical idea was used to initiate the song writing process. The process of recording, considering ideas and discussing the direction of the song, and then ultimately developing the song continued for approximately 5 hours. The song-writing process continued in this way until it was considered to be impossible to add any value to the creation.

The participant, whilst clearly familiar with the process of songwriting, stated that they were viewing the songwriting session as an opportunity to try a "different approach" to song-writing, and to perhaps try to achieve a different sounding musical style from normal. The participant mentioned particular artists at the beginning of the session and suggesting their intention to develop music informed by these influences. From the initial discussion a short musical idea was created or presented which would serve as the foundation for the song that would develop during the session.

The session was then transcribed in NVIVO for analysis. Observation of the video and statistical analysis was carried out with a range of initial, high level, analysis strategies considered. Some patterns in the data were observed.

\subsubsection{All Participants}

A further four studies were completed with participants from a range of backgrounds. From observing the results of this analysis, similarities were evident between the behaviors of each participant.

The participants, it was found, would regularly use musical influences as a way to communicate ideas. They would regularly reference the work of artists that they wished to emulate, or that the musical work reminded them of.

It was also found that participants would periodically enter into more intensive discussions about certain parts of the song. These discussions would, it appeared, make a significant change to the creative direction of the song. These observations were not analyzed statistically at this point. It was considered that a statistical analysis should be carried out in future.

In each case the participants stated that the approach that they had in mind for the session, before going into the studio, would be different to their usual approach. This may have implications related to each participant's intrinsic motivation. For example, as the musicians were writing for experimental reasons, with no intention of using the material in a commercial or professional capacity, they tended to take the opportunity to try a new tactic.

\section{Working Model of Song Writing Collaboration}

The emergent model developed from the sessions showed some definite similarities to the Systems Model of Creativity as. The use of anecdotal information in order to communicate ideas suggested that the participants were referencing a field and domain of established knowledge. This process also appeared to serve as a broad metric of quality standards, values, and rules. The sessions resulted in some behavior that could be described as similar to the 'Four-C Model'. Specifically, periods of creative development whilst a learning process was taking place. This type of learning took place predominantly at the beginning of the session when the initial ideas were being discussed. The data was then used to produce a basic model of the processes shown in Figure 1.

\section{Discussion}

The auto ethnographic study provided a large amount of data to analyze. Whilst the analysis has not yet been fully completed, early indications show that there are cases of repeated patterns in behaviours, and common methods of communication between participants. Many of the participants viewed the sessions as an opportunity to try a different approach to song creation. It was considered that this could be a further reason, outside of the original remit of the project, to carry out the research.

The wider scope of the research was to develop a collaborative artificial intelligence system capable of working with a musician to produce music. It was considered that data gathered during the auto ethnographic study would create the foundation of such a system.

\section{REFERENCES}

[1] A. Leyshon. 2009. The Software Slump?: digital music, the democratisation of technology, and the decline of the recording studio sector within the musical economy. Environment and Planning A, 41(6), pp.1309-1331.

[2] J. Bennett. 2011. Collaborative songwriting-the ontology of negotiated creativity in popular music studio practice. Journal on the Art of Record Production, (5).

[3] R. Fox and A. Khan. 2013. Artificial intelligence approaches to music composition. In Proceedings on the International Conference on Artificial Intelligence (ICAI) (p. 1). The Steering Committee of The World Congress in Computer Science, Computer Engineering and Applied Computing (WorldComp)

[4] V. Tran. 2009. Music Composition using Artificial Intelligence. Villanova, Pa. Villanova University.

[5] I. Steadman. 2012. Brian Eno on music that thinks for itself. Wired Magazine. Available at: https://www.wired.co.uk/article/brian-eno-peter-chilvers-scape (Accessed 8th June 2018).

[6] D. Sanchez. 2018. My Future Songwriting Career Just Got Deleted by an AI Startup. Digital Music News, https://www.digitalmusicnews.com/2018/03/22/amper-music-ai-composers/ (Accessed 22 ${ }^{\text {nd }}$ March 2018)

[7] Sony Computer Science Laboratories. Flow Machines. Available at: http://www.flow-machines.com/ (Accessed $8^{\text {th }}$ June 2018)

[8] E. Miranda 2002. Composing Music with Computers p187-9. Focal Press.

[9] C. Csikszentmihalyi. 2002. Flow: The Psychology of Happiness. Random House E-Books, Rider, Harper \& Row p39

[10] C. Csikzentmiyhayli, 2014. The Systems Model of Creativity - The Collected Works of Mihayli Csikzentmiyhayli. Springer E-Book. Springer Dordrecht Heidelberg New York London.

[11] R. Beghetto., J. Kaufman. 2009. Beyond Big and Little: The Four C Model of Creativity. Review of General Psychology 2009 Vol 13, No. 1, 1-12. 


\section{Initial Discussion}
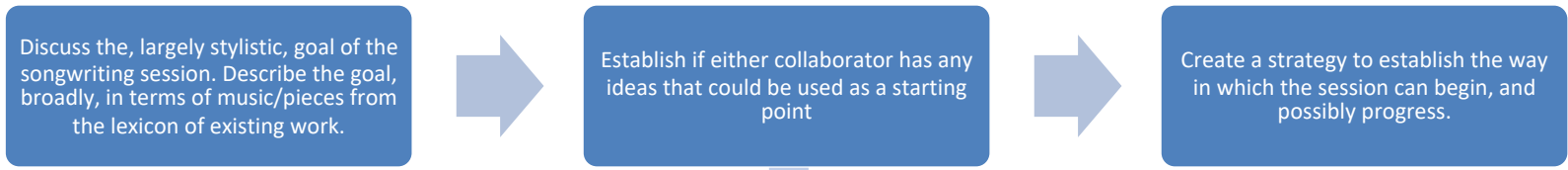

Learning and development of initial idea

Listen to initial idea

generated by the

participant/collaborator

Establish whether to

move to the next stage

of the creative process

or to try a different idea.

\section{Creative cycle}

Establish whether the

idea is in a form which

would allow further

development

Appraise the idea in it's

current form
Develop a way to create

a basis/platform from

which to record, edit,

and develop the idea

\section{Conclusion}

Critical appraisal of song up to the current point in time.
Establish whether song is finished because there are no more ideas, or it is considered to be completed.

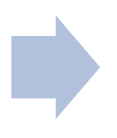

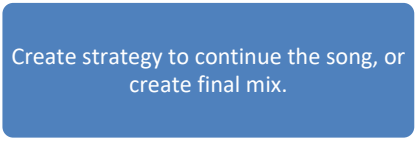

Figure 1: Flow of information from the song-writing process as observed during the auto-ethnographic study. 